\title{
Asymmetric Fermi surfaces for magnetic Schrödinger operators
}

\section{Report}

Author(s):

Feldman, Joel S.; Trubowitz, Eugene; Knörrer, Horst

Publication date:

1998

Permanent link:

https://doi.org/10.3929/ethz-a-004353129

Rights / license:

In Copyright - Non-Commercial Use Permitted

Originally published in:

FIM's preprints 


\title{
Asymmetric Fermi Surfaces for Magnetic Schrödinger Operators
}

\author{
Joel Feldman* \\ Department of Mathematics \\ University of British Columbia \\ Vancouver, B.C. \\ CANADA V6T $1 \mathrm{Z2}$ \\ Horst Knörrer, Eugene Trubowitz \\ Mathematik \\ ETH-Zentrum \\ CH-8092 Zürich \\ SWITZERLAND
}

\begin{abstract}
We consider Schrödinger operators with periodic magnetic field having zero flux through a fundamental cell of the period lattice. We show that, for a generic small magnetic field and a generic small Fermi energy, the corresponding Fermi surface is convex and not invariant under inversion in any point.
\end{abstract}

* Research supported in part by the Natural Sciences and Engineering Research Council of Canada, the Schweizerischer Nationalfonds zur Förderung der wissenschaftlichen Forschung and the Forschungsinstitut für Mathematik, ETH Zürich 


\section{Introduction}

Let $\Gamma$ be a lattice in $\mathbb{R}^{d}, d \geq 2$ and let $r>d$. Define

$$
\begin{aligned}
& \mathcal{A}=\left\{A=\left(A_{1}, \cdots, A_{d}\right) \in\left(L_{\mathbb{R}}^{r}\left(\mathbb{R}^{d} / \Gamma\right)\right)^{d} \mid \int_{\mathbb{R}^{d} / \Gamma} A(x) d x=0\right\} \\
& \mathcal{V}=\left\{V \in L_{\mathbb{R}}^{r / 2}\left(\mathbb{R}^{d} / \Gamma\right) \mid \int_{\mathbb{R}^{d} / \Gamma} V(x) d x=0\right\}
\end{aligned}
$$

For $(A, V) \in \mathcal{A} \times \mathcal{V}$ set

$$
H_{k}(A, V)=(i \nabla+A(x)-k)^{2}+V(x)
$$

When $d=2,3$, the operator $H_{k}(A, V)$ describes an electron in $\mathbb{R}^{d}$ with quasimomentum $k$ moving under the influence of the magnetic field with periodic vector potential $A(x)=$ $\left(A_{1}(x), \cdots A_{d}(x)\right)$ and electric field with periodic potential $V(x)$.

In general let

$$
e_{1}(k, A, V) \leq e_{2}(k, A, V) \leq \cdots
$$

be the eigenvalues of the operator $H_{k}(A, V)$ on $L^{2}\left(\mathbb{R}^{2} / \Gamma\right)$. The restriction of $e_{n}(k, A, V)$ to the first Brillouin zone $\mathcal{B}$ of $\Gamma$ is called the $n$-th band function of $A$. Observe that

$$
e_{1}(k, 0,0)=|k|^{2}
$$

The Fermi surface of $(A, V)$ with energy $\lambda$ is defined as

$$
F_{\lambda}(A, V)=\left\{k \in \mathcal{B} \mid e_{n}(k, A)=\lambda \text { for some } n\right\}
$$

Because $\overline{H_{k}(A, V)}=H_{-k}(-A, V)$

$$
e_{n}(-k,-A, V)=e_{n}(k, A, V)
$$

for all $n \geq 1$. In particular, when $A=0$,

$$
e_{n}(-k, 0, V)=e_{n}(k, 0, V)
$$

for all $n \geq 1$, so that $F_{\lambda}(0, V)=-F_{\lambda}(0, V)$ for all $\lambda$ and $V$. For all $(A, V) \in \mathcal{A} \times \mathcal{V}, \lambda \in \mathbb{R}$ and $p \in \mathbb{R}^{d}$

$$
p-F_{\lambda}(A, V)=\left\{p-k \mid k \in F_{\lambda}(A, V)\right\}
$$

The main result of this paper is 
Theorem There is a neighbourhood $\mathcal{A}_{0} \times \mathcal{V}_{0}$ of the origin in $\mathcal{A} \times \mathcal{V}$ and $\lambda_{0}>0$ such that

(i) for all $(\lambda, a, V) \in\left(-\infty, \lambda_{0}\right) \times \mathcal{A}_{0} \times \mathcal{V}_{0}, \quad F_{\lambda}(A, V)$ is either a strictly convex $(d-1)-$ dimensional real analytic submanifold of $\mathcal{B}$, or consists of one point, or is empty.

(ii) there is an open dense subset $\mathcal{S}$ of $\left(-\infty, \lambda_{0}\right) \times \mathcal{A}_{0} \times \mathcal{V}_{0}$ such that for all $(\lambda, A, V) \in \mathcal{S}$ and all $p \in \mathbb{R}^{d}$

$$
F_{\lambda}(A, V) \cap\left(p-F_{\lambda}(A, V)\right)
$$

has dimension at most $d-2$. Furthermore $\mathcal{S} \cap\left(\left(-\infty, \lambda_{0}\right) \times \mathcal{A}_{0} \times\{0\}\right)$ is open and dense in $\left(-\infty, \lambda_{0}\right) \times \mathcal{A}_{0} \times\{0\}$.

The Theorem shows that, for generic small periodic magnetic fields of mean zero and generic small Fermi energies, the Fermi surface is strictly convex and does not have inversion symmetry about any point. In particular, when $d=2$, the intersection of the Fermi surface and its inversion in any point is generically a finite set of points. The same statements hold if one considers both electric and magnetic fields. This inversion asymmetry suppresses the Cooper channel in weakly-coupled short-range many Fermion systems. See [FKLT].

There are real materials containing a periodic array of magnetized ions with zero flux through a fundamental cell. See the review [O, pp 1-48].

In $\oint$ II we show that the Fermi surface is always a real analytic subvariety of $\mathbb{R}^{d}$, and that it depends holomorphically on $(A, V) \in \mathcal{A} \times \mathcal{V}$. The proof of the main Theorem, presented in $\S I I I$, is based on a third order perturbation calculation around $(A, V)=(0,0)$. 


\section{Analyticity of the Fermi surfaces}

Let

$$
\begin{aligned}
& \mathcal{A}_{\mathbb{C}}=\left\{A=\left(A_{1}, \cdots, A_{d}\right) \in\left(L^{r}\left(\mathbb{R}^{d} / \Gamma\right)\right)^{d} \mid \int_{\mathbb{R}^{d} / \Gamma} A(x) d x=0\right\} \\
& \mathcal{V}_{\mathbb{C}}=\left\{V \in L^{r / 2}\left(\mathbb{R}^{d} / \Gamma\right) \mid \int_{\mathbb{R}^{d} / \Gamma} V(x) d x=0\right\}
\end{aligned}
$$

be the complexifications of $\mathcal{A}$ resp. $\mathcal{V}$.

Theorem. There exists an analytic function $F$ on $\mathbb{C}^{d} \times \mathbb{C} \times \mathcal{A}_{\mathbb{C}} \times \mathcal{V}_{\mathbb{C}}$ such that, for $k, A, V$ real,

$$
\lambda \in \operatorname{Spec}\left(H_{k}(A, V)\right) \quad \Longleftrightarrow \quad F(k, \lambda, A, V)=0
$$

Corollary Fix an open ball $D$ in the first Brillouin zone $\mathcal{B}$ that contains 0 such that $\bar{D} \subset \mathcal{B}^{\circ}$. There is a neighbourhood $U$ of the origin in $\mathcal{A} \times \mathcal{V}$ and there is $\lambda_{0}>0$ such that

i) For all $(A, V) \in U$ and all $k \in D$

$$
e_{1}(k, A, V)<e_{2}(k, A, V)
$$

ii) The map

$$
D \times U \longrightarrow \mathbb{R}, \quad(k, A, V) \longmapsto e_{1}(k, A, V)
$$

is real analytic.

iii) For each fixed $(A, V) \in U$ the Hessian of the map $k \longmapsto e_{1}(k, A, V)$ is positive definite. Furthermore $\inf _{k \in D} e_{1}(k, A, V)<\lambda_{0}$.

iv) For each $(A, V) \in U$ and each $\lambda<\lambda_{0}$ the Fermi surface $F_{\lambda}(A, V)$ is either empty, or consists of one point only, or is a real analytic smooth strictly convex $(d-1)$-dimensional real analytic manifold that is completely contained in $D$.

Proof of the Corollary: The spectrum of $H_{k}(0,0)$ is $\left\{|k+b|^{2} \mid b \in \Gamma^{\sharp}\right\}$. Hence, for $k \in D, e_{1}(k, 0,0)=|k|^{2}$ and $e_{2}(k, 0,0)>e_{1}(k, 0,0)$. The Corollary follows from repeated application of the Implicit Function Theorem and continuity. 
Proof of the Theorem: Write

$$
(i \nabla+A(x)-k)^{2}+V(x)-\lambda=1-\Delta+u(k, \lambda)+w(k, A, V)
$$

with

$$
\begin{aligned}
u(k, \lambda) & =-2 i k \cdot \nabla+k^{2}-\lambda-11 \\
w(k, A, V) & =i \nabla \cdot A+i A \cdot \nabla-2 k \cdot A+A^{2}+V
\end{aligned}
$$

At the end of this Section we prove

Lemma. Let $\|B\|_{r}=\left[\operatorname{tr}\left(B^{*} B\right)^{r / 2}\right]^{1 / r}$ where $\operatorname{tr}$ denotes the trace defined on trace class operators on $L^{2}\left(\mathbb{R}^{d} / \Gamma\right)$. There is a constant const ${ }_{\Gamma, r, d}$ such that

a)

$$
\begin{gathered}
\left\|\frac{1}{\sqrt{1-\Delta}} w(k, A, V) \frac{1}{\sqrt{1-\Delta}}\right\|_{r} \leq \operatorname{const}_{\Gamma, r, d}\left((1+|k|)\|A\|_{L^{r}}+\|A\|_{L^{r}}^{2}+\|V\|_{L^{r / 2}}\right) \\
\left\|\frac{1}{\sqrt{1-\Delta}} u(k, \lambda) \frac{1}{\sqrt{1-\Delta}}\right\|_{r} \leq \operatorname{const}_{\Gamma, r, d}\left(1+|k|^{2}+|\lambda|\right)
\end{gathered}
$$

b)

c) Let $0 \leq \epsilon \leq \frac{r-d}{2 r}$. There is a constant const $_{\Gamma, r, d, k, \lambda, A, V}$ such that

$$
\begin{aligned}
|\langle(u(k, \lambda)+w(k, A, V)) \phi, \psi\rangle| \leq \operatorname{const}_{\Gamma, r, d, k, \lambda, A, V} & \left(\left\|(\mathbb{1}-\Delta)^{(1-\epsilon) / 2} \phi\right\|\left\|(\mathbb{1}-\Delta)^{1 / 2} \psi\right\|\right. \\
& \left.+\left\|(\mathbb{1}-\Delta)^{1 / 2} \phi\right\|\left\|(\mathbb{1}-\Delta)^{(1-\epsilon) / 2} \psi\right\|\right)
\end{aligned}
$$

for all $\psi, \phi \in L^{2}\left(\mathbb{R}^{d} / \Gamma\right)$.

Because $L^{s}\left(\mathbb{R}^{d} / \Gamma\right) \supset L^{s^{\prime}}\left(\mathbb{R}^{d} / \Gamma\right)$ for all $1 \leq s \leq s^{\prime}$, we may assume, without loss of generality, that $r \leq d+1$. Then the Lemma implies that

$$
F(k, \lambda, A, V)=\operatorname{det}_{d+1}\left(1+\frac{1}{\sqrt{1-\Delta}} u(k, \lambda) \frac{1}{\sqrt{1-\Delta}}+\frac{1}{\sqrt{1-\Delta}} w(k, A, V) \frac{1}{\sqrt{1-\Delta}}\right)
$$

is a well-defined analytic function on $\mathbb{C}^{d} \times \mathbb{C} \times \mathcal{A}_{\mathbb{C}} \times \mathcal{V}_{\mathbb{C}}$. Here, $\operatorname{det}_{d+1}(\mathbb{1}+B)$ is the regularized determinant which, for matrices, is defined by

$$
\operatorname{det}_{d+1}(\mathbb{1}+B)=\exp \left(\sum_{i=1}^{d} \frac{(-1)^{i}}{i} \operatorname{tr} B^{i}\right) \operatorname{det}(11+B)
$$

This regularized determinant is defined for $B$ with $\|B\|_{d+1}$ finite. See [S], Theorem 9.2. It is analytic since one can take limits of finite rank approximations of $B$. 
Let $\mathcal{D}$ be the domain of $\sqrt{1-\Delta}$. By the Lemma,

$$
(i \nabla+A(x)-k)^{2}+V(x)-\lambda=11-\Delta+u(k, \lambda)+w(k, A, V)
$$

gives a well-defined quadratic form on $\mathcal{D} \times \mathcal{D}$. Furthermore, for $\phi \in \mathcal{D}$

$$
\langle(1-\Delta+u(k, \lambda)+w(k, A, V)) \phi, \phi\rangle \geq\|\sqrt{1-\Delta} \phi\|^{2}-\text { const }\left\|(\mathbb{1}-\Delta)^{(1-\epsilon) / 2} \phi\right\|\|\sqrt{\mathbb{1}-\Delta} \phi\|
$$

For any $\delta>0$ there is a constant $c_{\delta}$ such that

$$
\left\|(1-\Delta)^{(1-\epsilon) / 2} \phi\right\| \leq \delta\|\sqrt{1-\Delta} \phi\|+c_{\delta}\|\phi\|
$$

Choosing $\delta$ appropriately,

$$
\begin{aligned}
\langle(1-\Delta+u(k, \lambda)+w(k, A, V)) \phi, \phi\rangle & \geq \frac{1}{2}\|\sqrt{1-\Delta} \phi\|^{2}-\operatorname{const} c_{\delta}\|\phi\|\|\sqrt{1-\Delta} \phi\| \\
& \geq-\frac{1}{2}\left(\operatorname{const} c_{\delta}\|\phi\|\right)^{2}
\end{aligned}
$$

and the form is semibounded. It is closed since

$$
\frac{1}{4}\|\sqrt{1-\Delta} \phi\|^{2}-\mathrm{const}\|\phi\|^{2} \leq|\langle(1-\Delta+u(k, \lambda)+w(k, A, V)) \phi, \phi\rangle| \leq \mathrm{const}\|\sqrt{1-\Delta} \phi\|^{2}
$$

Hence there is a unique associated self adjoint semibounded operator $H_{k}(A, V)$.

The resolvent $(1-\Delta)^{-1}$ is compact. Hence by the resolvent identity and part a) of the Lemma the resolvent of $H_{k}(A, V)$ is also compact. Hence the spectrum of $H_{k}(A, V)$ is discrete. Then $\lambda \in \operatorname{Spec}\left(H_{k}(A, V)\right)$ if and only if there exists $\psi \in \mathcal{D}_{H_{k}(A, V)} \subset \mathcal{D}$ such that

$$
\left(H_{k}(A, V)-\lambda\right) \frac{1}{\sqrt{1-\Delta}} \sqrt{11-\Delta} \psi=0
$$

This is the case if and only if $\frac{1}{\sqrt{1-\Delta}}\left(H_{k}(A, V)-\lambda\right) \frac{1}{\sqrt{1-\Delta}}$ has a nontrivial kernel. By [S], Theorem 9.2 (e), this is the case if and only if $F(k, \lambda, A, V)=0$.

Proof of the Lemma: a) We repeatedly apply the result that, for any $r \geq 2$ and any $f \in \ell^{r}\left(\Gamma^{\#}\right)$ and $g \in L^{r}\left(\mathbb{R}^{d} / \Gamma\right)$,

$$
\|f(i \nabla) g(x)\| \leq \operatorname{vol}\left(\mathbb{R}^{d} / \Gamma\right)^{-1 / r}\|f\|_{\ell^{r}\left(\Gamma^{\#}\right)}\|g\|_{L^{r}\left(\mathbb{R}^{d} / \Gamma\right)}
$$


This is proven just as in [S] Theorem 4.1, the corresponding bound for operators on $L^{2}\left(\mathbb{R}^{d}\right)$. One first proves easily that the Hilbert-Schmidt norm of $f(i \nabla) g(x)$ is bounded by $\operatorname{vol}\left(\mathbb{R}^{d} / \Gamma\right)^{-1 / 2}\|f\|_{\ell^{2}\left(\Gamma^{\#}\right)}\|g\|_{L^{2}\left(\mathbb{R}^{d} / \Gamma\right)}$ and that the operator norm of $f(i \nabla) g(x)$ is bounded by $\|f\|_{\ell^{\infty}\left(\Gamma^{\#}\right)}\|g\|_{L^{\infty}\left(\mathbb{R}^{d} / \Gamma\right)}$. One then interpolates using $[\mathrm{S}]$, Theorem 2.9.

As the operator norms $\left\|\frac{1}{\sqrt{\mathbb{1}-\Delta}} \nabla\right\| \leq 1,\left\|\frac{1}{\sqrt{\mathbb{1}-\Delta}} k\right\| \leq|k|$ and $\left\|B B^{\prime}\right\|_{r} \leq\|B\|_{r}\left\|B^{\prime}\right\|$, we have

$$
\left\|\frac{1}{\sqrt{1-\Delta}} w \frac{1}{\sqrt{1-\Delta}}\right\|_{r} \leq(2+|k|)\left\|\frac{1}{\sqrt{1-\Delta}} a\right\|_{r}+\left\|\frac{1}{\sqrt{1-\Delta}} A\right\|_{r}^{2}+\left\|\frac{1}{\sqrt{1-\Delta}} V \frac{1}{\sqrt{1-\Delta}}\right\|_{r}
$$

Write

$$
\frac{1}{\sqrt{1-\Delta}}=f(i \nabla) \quad \text { with } \quad f(b)= \begin{cases}1 & \text { if } \mathrm{b}=0 \\ \frac{1}{\sqrt{1+b^{2}}} & \text { if } b \neq 0\end{cases}
$$

This $f \in \ell^{r}\left(\Gamma^{\#}\right)$ for all $r>d$, so the desired result follows from $(*)$ with $g=A_{1}, \cdots, A_{d}, \sqrt{V}$.

b) The spectrum of $\frac{1}{\sqrt{1-\Delta}} u(k, \lambda) \frac{1}{\sqrt{1-\Delta}}$ is

$$
\left\{\frac{2 k \cdot b+k^{2}-\lambda-1}{1+b^{2}} \mid b \in \Gamma^{\#}\right\}
$$

For any $r>d$, the $\ell^{r}\left(\Gamma^{\#}\right)-$ norm of $\frac{2 k \cdot b+k^{2}-\lambda-1}{1+b^{2}}$, which is also the $\|\cdot\|_{r}$ norm of $\frac{1}{\sqrt{\mathbb{1}-\Delta}} u(k, \lambda) \frac{1}{\sqrt{\mathbb{1}-\Delta}}$, is bounded by const $_{\Gamma, r}\left(1+|k|^{2}+|\lambda|\right)$.

c) Denote $D=\sqrt{(1-\Delta)}$. The condition on $\epsilon$ implies that $r(1-\epsilon) \geq d+\frac{r-d}{2}>d$ so that $\frac{1}{\left(1+b^{2}\right)^{r(1-\epsilon) / 2}}$ is still summable. So, as in part a),

$$
\begin{aligned}
\left\|\frac{1}{D^{1-\epsilon}} A\right\| & \leq \text { const }_{\Gamma, r}\|a\|_{L^{r}} \\
\left\|\frac{1}{D^{1-\epsilon}} V \frac{1}{D}\right\| & \leq \operatorname{const}_{\Gamma, r}\|V\|_{L^{r / 2}} \\
\left\|u \frac{1}{D}\right\| & \leq \operatorname{const}_{\Gamma, r}\left(1+|k|^{2}+|\lambda|\right)
\end{aligned}
$$

Consequently,

$$
\begin{aligned}
|\langle(i \nabla A) \phi, \psi\rangle| & \leq \text { const }_{\Gamma, r}\|A\|_{L^{r}}\left\|D^{1-\epsilon} \phi\right\|\|D \psi\| \\
|\langle(A i \nabla) \phi, \psi\rangle| & \leq \text { const }_{\Gamma, r}\|a\|_{L^{r}}\|D \phi\|\left\|D^{1-\epsilon} \psi\right\| \\
|\langle(2 k \cdot A) \phi, \psi\rangle| & \leq \text { const }_{\Gamma, r}|k|\|A\|_{L^{r}}\left\|D^{1-\epsilon} \phi\right\|\|\psi\| \\
|\langle(A \cdot A) \phi, \psi\rangle| & \leq \text { const }_{\Gamma, r}\|A\|_{L^{r}}^{2}\left\|D^{1-\epsilon} \phi\right\|\left\|D^{1-\epsilon} \psi\right\| \\
|\langle V \phi, \psi\rangle| & \leq \text { const }_{\Gamma, r}\|V\|_{L^{r / 2}}\left\|D^{1-\epsilon} \phi\right\|\|D \psi\| \\
|\langle u \phi, \psi\rangle| & \leq \text { const }_{\Gamma, r}\left(1+|k|^{2}+|\lambda|\right)\|\phi\|\|D \psi\|
\end{aligned}
$$




\section{Proof of the Main Theorem}

For simplicity we write $e(k, A, V)=e_{1}(k, A, V)$. By part (iii) of the Corollary in $\S I I$, for each $(A, V) \in U$ the band function $e(-, A, V)$ has a unique extremum $k_{m i n}(A, V)$. This extremum is a nondegenerate minimum. It follows from the implicit function theorem that $k_{\text {min }}(A, V)$ depends analytically on $(A, V)$. The same is true for the corresponding critical value $\lambda_{\min }(A, V)=e\left(k_{\min }(A, V), A, V\right)$. Observe that $\lambda_{\min }(A, V)<\lambda_{0}$ by part (iii) of the Corollary in $\S I I$. We set

$$
P=\left\{(\lambda, A, V) \in \mathbb{R} \times U \mid \lambda_{\min }(A, V)<\lambda<\lambda_{0}\right\}
$$

Then for each $(\lambda, A, V) \in P$ the Fermi surface $F_{\lambda}(A, V)$ is a smooth, real analytic, strictly convex $(d-1)$-dimensional manifold which is not empty. For $k \in F_{\lambda}(A, V)$ denote by $n(k)$ the outward unit normal vector to $F_{\lambda}(A, V)$ at $\mathrm{k}$. If $(\lambda, A, V) \in P$ then for each $\xi$ on the unit sphere $S^{d-1}$ there is a unique point $k_{\lambda}(\xi, A, V) \in F_{\lambda}(A, V)$ such that $n\left(k_{\lambda}(\xi, A, V)\right)=\xi$. Again it follows from the implicit function theorem that

$$
S^{d-1} \times P \longrightarrow D, \quad(\xi, \lambda, A, V) \longmapsto k_{\lambda}(\xi, A, V)
$$

is a real analytic map.

To prove the Theorem stated in the Introduction we have to show that for $(\lambda, A, V)$ in an open dense subset of $P$ and all $p \in \mathbb{R}^{d}$ the intersection $F_{\lambda}(A, V) \cap\left(p-F_{\lambda}(A, V)\right)$ has dimension at most $d-2$. Since for all $(\lambda, A, V) \in P$ the manifold $F_{\lambda}(A, V)$ is real analytic, smooth and strictly convex, one has for all $(\lambda, A, V) \in P$ and all $p \in \mathbb{R}^{d}$

$$
\operatorname{dim}\left(F_{\lambda}(A, V) \cap\left(p-F_{\lambda}(A, V)\right)\right) \leq d-2 \quad \text { or } \quad F_{\lambda}(A, V)=\left(p-F_{\lambda}(A, V)\right)
$$

If $F_{\lambda}(A, V)=\left(p-F_{\lambda}(A, V)\right)$ then inversion in the point $p / 2$ maps the point of $F_{\lambda}(A, V)$ with normal vector $\xi$ to the point of $F_{\lambda}(A, V)$ with normal vector $-\xi$. In other words,

$$
F_{\lambda}(A, V)=\left(p-F_{\lambda}(A, V)\right) \quad \Longrightarrow \quad k_{\lambda}(\xi, A, V)+k_{\lambda}(-\xi, A, V)=p \quad \text { for all } \xi \in S^{d-1}
$$

Therefore the set of all $(\lambda, A, V) \in P$ for which there is a point $p \in \mathbb{R}^{d}$ such that $\operatorname{dim}\left(F_{\lambda}(A, V) \cap\left(p-F_{\lambda}(A, V)\right)\right)>d-2$ is contained in

$$
\mathcal{S}^{\prime}=\left\{(\lambda, A, V) \in P \mid \nabla_{\xi}\left(k_{\lambda}(\xi, A, V)+k_{\lambda}(-\xi, A, V)\right)=0 \text { for all } \xi \in S^{d-1}\right\}
$$


Observe that $\mathcal{S}^{\prime}$ is the intersection of the analytic hypersurfaces

$$
\left\{(\lambda, A, V) \in P \mid \nabla_{\xi}\left(k_{\lambda}(\xi, A, V)+k_{\lambda}(-\xi, A, V)\right)=0\right\}, \quad \xi \in S^{d-1}
$$

Thus to show that the complement of $\mathcal{S}^{\prime}$ is open and dense it suffices to exhibit one triple $(\lambda, A, V) \in P$ that does not lie in $\mathcal{S}^{\prime}$. We will do this by choosing $V=0$, choosing a particular two dimensional vector potential $A$ and showing that for small $t$ and appropriate $\lambda$ the triple $(\lambda, t \cdot A, 0)$ does not lie in $\mathcal{S}^{\prime}$. This then also shows that the complement of $\mathcal{S}^{\prime} \cap\{(\lambda, A, V) \in P \mid V=0\}$ is open and dense in $\{(\lambda, A, V) \in P \mid V=0\}$.

In the following calculation we will only consider the points $(\lambda, t \cdot A, 0)$ of $P$ with a particular two dimensional vector potential $A$. Therefore we restrict ourselves to the case $d=2$ and delete the $V$-variable in the notation. First we compute the first three $t$ derivatives of $e(k, t \cdot A)$ at the origin for arbitrary (two-dimensional) $A$. We use the notation $\dot{f}(k)=\left.\frac{d}{d t} f(k, t)\right|_{t=0}$.

Lemma. Fix $A \in \mathcal{A}$. Put

$$
\epsilon(k, t)=e(k, t \cdot A)
$$

Then there is a constant $C$ such that for all $k \in D$

$$
\begin{aligned}
\dot{\epsilon}(k)= & 0 \\
\ddot{\epsilon}(k)= & C-2 \sum_{b \in \Gamma^{\#} \backslash\{0\}} \frac{1}{b^{2}+2 k \cdot b}|(2 k+b) \cdot \hat{A}(b)|^{2} \\
\dddot{\epsilon}(k)= & 12 \operatorname{Re} \sum_{b, c \in \Gamma^{\#} \backslash\{0\}} \frac{1}{b^{2}+2 k \cdot b}[\hat{A}(-c) \cdot \hat{A}(c-b)][(2 k+b) \cdot \hat{A}(b)] \\
& -6 \sum_{b, c \in \Gamma^{\#} \backslash\{0\}} \frac{[(2 k+c) \cdot \hat{A}(-c)]}{c^{2}+2 k \cdot c}[(2 k+b+c) \cdot \hat{A}(c-b)] \frac{[(2 k+b) \cdot \hat{A}(b)]}{b^{2}+2 k \cdot b}
\end{aligned}
$$

Here $\hat{A}(b)=\left(\hat{A}_{1}(b), \hat{A}_{2}(b)\right)$ are the Fourier coefficients of $A$, i.e

$$
A(x)=\sum_{b \in \Gamma^{\#}} \hat{A}(b) e^{i b \cdot x}
$$

and

$$
\Gamma^{\#}=\left\{b \in \mathbb{R}^{2} \mid<b, \gamma>\in 2 \pi \mathbb{Z} \text { for all } \gamma \in \Gamma\right\}
$$


is the dual lattice to $\Gamma$. Furthermore for each $\lambda \in\left(0, \lambda_{0}\right)$ and every $\xi \in S^{1}$

$$
\begin{aligned}
\left.\frac{d}{d t} k_{\lambda}(\xi, t \cdot A)\right|_{t=0} & =0 \\
\left.2 \sqrt{\lambda} \xi \cdot \frac{d^{2}}{d t^{2}} k_{\lambda}(\xi, t \cdot A)\right|_{t=0} & =-\ddot{\epsilon}(\sqrt{\lambda} \xi) \\
\left.2 \sqrt{\lambda} \xi \cdot \frac{d^{3}}{d t^{3}} k_{\lambda}(\xi, t \cdot A)\right|_{t=0} & =-\dddot{\epsilon}(\sqrt{\lambda} \xi)
\end{aligned}
$$

Proof: Let $\psi_{k}(t)$ be the eigenfunction of eigenvalue $\epsilon(k, t)$ for the operator $H_{k}(t \cdot A)$ normalized by the conditions that

$$
\psi_{k}(0)=\frac{1}{\sqrt{\mathrm{vol}}}
$$

where vol is the volume of $\mathbb{R}^{2} / \Gamma$, and

$$
<\psi_{k}(0), \psi_{k}(t)>=1
$$

for all $t$. Then, for small $t$ and $k \in D, \psi_{k}(t)$ is an analytic function of $t$ and $k$. For convenience we suppress the argument $k$ in the following computation.

From the identity

$$
H \psi=\epsilon \psi
$$

one gets by differentiation

$$
\begin{aligned}
\dot{H} \psi+H \dot{\psi} & =\dot{\epsilon} \psi+\epsilon \dot{\psi} \\
\ddot{H} \psi+2 \dot{H} \dot{\psi}+H \ddot{\psi} & =\ddot{\epsilon} \psi+2 \dot{\epsilon} \dot{\psi}+\epsilon \ddot{\psi} \\
\dddot{H} \psi+3 \ddot{H} \dot{\psi}+3 \dot{H} \ddot{\psi}+H \ddot{\psi} & =\dddot{\epsilon} \psi+3 \ddot{\epsilon} \dot{\psi}+3 \dot{\epsilon} \ddot{\psi}+\epsilon \ddot{\psi}
\end{aligned}
$$

Forming the inner product of these equations with $\psi(0)$ gives

$$
\begin{aligned}
\dot{\epsilon} & =<\dot{H} \psi, \psi> \\
\ddot{\epsilon} & =<\ddot{H} \psi, \psi>+2<\dot{H} \dot{\psi}, \psi> \\
\dddot{\epsilon} & =<\ddot{H} \psi, \psi>+3<\ddot{H} \dot{\psi}, \psi>+3<\dot{H} \ddot{\psi}, \psi>
\end{aligned}
$$


since $\langle\dot{\psi}, \psi>=<\ddot{\psi}, \psi>=<\dddot{\psi}, \psi>=0$ and $<H \dot{\psi}, \psi>=<H \ddot{\psi}, \psi>=<H \dddot{\psi}, \psi>=0$. However

$$
\begin{aligned}
\frac{d}{d t} H_{k}(t \cdot A) & =A \circ(i \nabla+t \cdot A-k)+(i \nabla+t \cdot A-k) \circ A \\
& =2 A \circ(i \nabla+t \cdot A-k)+i\left(\frac{\partial A_{1}}{\partial x_{1}}+\frac{\partial A_{2}}{\partial x_{2}}\right)
\end{aligned}
$$

Therefore for $t=0$

$$
\dot{H}_{k} \psi=\frac{1}{\sqrt{\mathrm{vol}}} \dot{H}_{k} 1=\frac{1}{\sqrt{\mathrm{vol}}}\left(-2 k \cdot A+i\left(\frac{\partial A_{1}}{\partial x_{1}}+\frac{\partial A_{2}}{\partial x_{2}}\right)\right)
$$

Taking inner product with $\psi(0)=\frac{1}{\sqrt{\text { vol }}} \cdot 1$ we get

$$
<\dot{H} \psi, \psi>=\frac{1}{\mathrm{vol}}\left\langle\left(-2 k \cdot A+i\left(\frac{\partial A_{1}}{\partial x_{1}}+\frac{\partial A_{2}}{\partial x_{2}}\right)\right), 1\right\rangle=0
$$

and hence

$$
\dot{\epsilon}=0
$$

From (1) we now deduce

$$
\dot{\psi}=-(H-\epsilon)^{-1} \dot{H} \psi
$$

where, by definition, $(H-\epsilon)^{-1}$ vanishes on $\psi(0)$ and is the inverse of $(H-\epsilon)$ when restricted to the orthogonal complement of $\psi(0)$. Substituting into (5)

$$
\ddot{\epsilon}=<\ddot{H} \psi, \psi>-2<\dot{H}(H-\epsilon)^{-1} \dot{H} \psi, \psi>
$$

Now by (7)

$$
\ddot{H}=2\left(A_{1}^{2}+A_{2}^{2}\right)
$$

so that $\langle\ddot{H} \psi, \psi\rangle$ is a constant $C=\frac{2}{\text { vol }}\left\langle\left(A_{1}^{2}+A_{2}^{2}\right), 1\right\rangle$ independent of $k$. Using (8) we therefore get

$$
\ddot{\epsilon}=C-\frac{2}{\operatorname{vol}}\left\langle\left(H_{k}-\epsilon\right)^{-1}\left(-2 k \cdot A+i\left(\frac{\partial A_{1}}{\partial x_{1}}+\frac{\partial A_{2}}{\partial x_{2}}\right)\right),\left(-2 k \cdot A+i\left(\frac{\partial A_{1}}{\partial x_{1}}+\frac{\partial A_{2}}{\partial x_{2}}\right)\right)\right\rangle
$$

Since

$$
\left(-2 k \cdot A+i\left(\frac{\partial A_{1}}{\partial x_{1}}+\frac{\partial A_{2}}{\partial x_{2}}\right)\right)=-\sum_{b \in \Gamma^{\#}}(2 k+b) \cdot \hat{A}(b) e^{i b \cdot x}
$$

and

$$
\left(H_{k}(0,0)-\epsilon(0)\right) e^{i b \cdot x}=\left(b^{2}+2 k \cdot b\right) e^{i b \cdot x}
$$


we get

$$
\begin{aligned}
\ddot{\epsilon} & =C-\frac{2}{\mathrm{vol}}\left\langle\sum_{b \in \Gamma^{\#} \backslash\{0\}} \frac{1}{b^{2}+2 k \cdot b}((2 k+b) \cdot \hat{A}(b)) e^{i b \cdot x}, \sum_{b \in \Gamma^{\#}}((2 k+b) \cdot \hat{A}(b)) e^{i b \cdot x}\right\rangle \\
& =C-2 \sum_{b \in \Gamma^{\#} \backslash\{0\}} \frac{1}{b^{2}+2 k \cdot b}|(2 k+b) \cdot \hat{A}(b)|^{2}
\end{aligned}
$$

From (2) we deduce that

$$
\begin{aligned}
\ddot{\psi} & =-(H-\epsilon)^{-1}(\ddot{H} \psi-\ddot{\epsilon} \psi+2 \dot{H} \dot{\psi}) \\
& =-(H-\epsilon)^{-1} \ddot{H} \psi+2(H-\epsilon)^{-1} \dot{H}(H-\epsilon)^{-1} \dot{H} \psi
\end{aligned}
$$

Using (6), (9), (10) and the fact that $\dddot{H}=0$, this gives

$$
\begin{aligned}
\dddot{\epsilon}=-3<\ddot{H}(H-\epsilon)^{-1} \dot{H} \psi, \psi>-3<\dot{H}(H-\epsilon)^{-1} \ddot{H} \psi, \psi> \\
\quad+6<\dot{H}(H-\epsilon)^{-1} \dot{H}(H-\epsilon)^{-1} \dot{H} \psi, \psi> \\
=-6 \operatorname{Re}<(H-\epsilon)^{-1} \dot{H} \psi, \ddot{H} \psi>+6<\dot{H}(H-\epsilon)^{-1} \dot{H} \psi,(H-\epsilon)^{-1} \dot{H} \psi>
\end{aligned}
$$

Since

$$
\begin{aligned}
(H-\epsilon)^{-1} \dot{H} \psi & =-\frac{1}{\sqrt{\mathrm{vol}}} \sum_{b \in \Gamma^{\#} \backslash\{0\}} \frac{1}{b^{2}+2 k \cdot b}((2 k+b) \cdot \hat{A}(b)) e^{i b \cdot x} \\
\ddot{H} \psi & =\frac{2}{\sqrt{\mathrm{vol}}} \sum_{b, c \in \Gamma^{\#}} \hat{A}(b-c) \cdot \hat{A}(c) e^{i b \cdot x} \\
\frac{1}{\mathrm{vol}}\left\langle\dot{H} e^{i b \cdot x}, e^{i c \cdot x}\right\rangle & =-(2 k+b+c) \cdot \hat{A}(c-b)
\end{aligned}
$$

we get

$$
\begin{aligned}
\dddot{\epsilon}= & 12 \operatorname{Re} \sum_{b, c \in \Gamma^{\#} \backslash\{0\}} \frac{1}{b^{2}+2 k \cdot b}[(2 k+b) \cdot \hat{A}(b)][\hat{A}(-c) \cdot \hat{A}(c-b)] \\
& -6 \sum_{b, c \in \Gamma^{\#} \backslash\{0\}} \frac{[(2 k+b) \cdot \hat{A}(-b)]}{b^{2}+2 k \cdot b}[(2 k+b+c) \cdot \hat{A}(c-b)] \frac{[(2 k+c) \cdot \hat{A}(c)]}{c^{2}+2 k \cdot c}
\end{aligned}
$$

This proves the statement about the derivatives of $\epsilon$.

We now prove the statement about the derivatives of $t \mapsto k_{\lambda}(\xi, t \cdot A)$ for fixed $\lambda \in\left(0, \lambda_{0}\right)$ and $\xi \in S^{1}$. To simplify notation put

$$
\kappa(\xi, t)=k_{\lambda}(\xi, t \cdot A)
$$


Differentiating the identity

$$
\epsilon(\kappa(\xi, t), t)=\lambda
$$

we get

$$
\nabla_{k} \epsilon(\kappa(\xi, t), t) \cdot \frac{\partial}{\partial t} \kappa(\xi, t)+\frac{\partial}{\partial t} \epsilon(\kappa(\xi, t), t)=0
$$

Since $\dot{\epsilon}=0$ and $\nabla_{k} \epsilon(\kappa(\xi, 0), 0)=2 \sqrt{\lambda} \xi$, setting $t=0$ gives

$$
\xi \cdot \dot{\kappa}(\xi)=0
$$

If $\xi^{\perp}$ denotes the vector $\left(-\xi_{2}, \xi_{1}\right)$ perpendicular to $\xi=\left(\xi_{1}, \xi_{2}\right)$, then by the definition of $k_{\lambda}$ we have $\xi^{\perp} \cdot \nabla_{k} \epsilon(\kappa(\xi, t), t)=0$. Differentiating this identity we get

$$
\xi^{\perp} \cdot\left(\operatorname{Hessian}(\epsilon) \cdot \dot{\kappa}(\xi)+\nabla_{k} \dot{\epsilon}\right)=0
$$

Since for $t=0$ we have Hessian $(\epsilon)=2 \times 1$ and $\dot{\epsilon}=0$, we get

$$
\xi^{\perp} \cdot \dot{\kappa}(\xi)=0
$$

Putting (12a) and (12b) together gives

$$
\dot{\kappa}=0
$$

Differentiating (11) again and setting $t=0$ gives

$$
\dot{\kappa}(\xi) \cdot \operatorname{Hessian}_{k}(\epsilon) \cdot \dot{\kappa}(\xi)+2 \nabla_{k} \dot{\epsilon}(\kappa(\xi, 0)) \cdot \dot{\kappa}(\xi)+2 \sqrt{\lambda} \xi \cdot \ddot{\kappa}(\xi)+\ddot{\epsilon}(\kappa(\xi, 0))=0
$$

Using (12) we get

$$
2 \sqrt{\lambda} \xi \cdot \ddot{\kappa}(\xi)=-\ddot{\epsilon}(\kappa(\xi, 0))=-\ddot{\epsilon}(\sqrt{\lambda} \xi)
$$

Differentiating (11) twice, setting $t=0$ and using $\dot{\kappa}=0$

$$
3 \nabla_{k} \dot{\epsilon}(\kappa(\xi, 0)) \cdot \ddot{\kappa}(\xi)+2 \sqrt{\lambda} \xi \cdot \dddot{\kappa}(\xi)+\dddot{\epsilon}(\kappa(\xi, 0))=0
$$

Since $\dot{\epsilon}=0$

$$
2 \sqrt{\lambda} \xi \cdot \dddot{\kappa}(\xi)=-\dddot{\epsilon}(\kappa(\xi, 0))=-\dddot{\epsilon}(\sqrt{\lambda} \xi)
$$


We now continue the proof of the main Theorem, and consider again a fixed vector potential $A$ and a fixed $\lambda \in\left(0, \lambda_{0}\right)$. If

$$
F_{\lambda}(t \cdot A)=\left(p(\lambda, t)-F_{\lambda}(t \cdot A)\right) \quad \text { for all small } t
$$

then

$$
k_{\lambda}(\xi, t \cdot A)+k_{\lambda}(-\xi, t \cdot A)=p(\lambda, t)
$$

for all $\xi \in S^{1}$ and all small $t$. Multiplying this equality with $2 \sqrt{\lambda} \xi$, differentiating three times with respect to $t$ and setting $t=0$ gives

$$
2 \sqrt{\lambda} \xi \cdot\left(\left.\frac{d^{3}}{d t^{3}} k_{\lambda}(\xi, t \cdot A)\right|_{t=0}+\left.\frac{d^{3}}{d t^{3}} k_{\lambda}(-\xi, t \cdot A)\right|_{t=0}\right)=2 \sqrt{\lambda} \xi \cdot \dddot{p}(\lambda)
$$

for all $\xi \in S^{1}$. By the previous Lemma, this is equivalent to

$$
\dddot{\epsilon}(-k)-\dddot{\epsilon}(k)=2 k \cdot \dddot{p}(\lambda) \quad \text { for all } k \text { with }|k|^{2}=\lambda
$$

or, since $\dddot{\epsilon}(-k)=-\dddot{\epsilon}(k)$, to

$$
\dddot{\epsilon}(k)=-k \cdot \dddot{p}(\lambda) \quad \text { for all } k \text { with }|k|^{2}=\lambda
$$

As said above, it suffices to specify one vector potential $A$ and one $\lambda \in\left(0, \lambda_{0}\right)$ such that (13) fails. We now give a concrete example of a vector potential $A$ for which (13) does not hold any $\lambda>0$. Fix a nonzero vector $d \in \Gamma^{\#}$. Without loss of generality we may assume that $d=(1,0)$. Let

$$
\hat{A}(d)=\hat{A}(-d)=\hat{A}(2 d)=\hat{A}(-2 d)=d^{\perp} \quad \text { and } \quad \hat{A}(b)=0 \text { for } b \neq \pm d, \pm 2 d
$$

where $d^{\perp}=(0,1)$. Then, by the previous Lemma,

$$
\begin{aligned}
& \dddot{\epsilon}(k)= 12 \operatorname{Re} \sum_{b, c \in \Gamma^{\#} \backslash\{0\}} \frac{1}{b^{2}+2 k \cdot b}[\hat{A}(-c) \cdot \hat{A}(c-b)][(2 k+b) \cdot \hat{A}(b)] \\
&-6 \sum_{\substack{b, c \in \Gamma^{\#} \backslash\{0\} \\
=}} \frac{[(2 k+c) \cdot \hat{A}(-c)]}{c^{2}+2 k \cdot c}[(2 k+b+c) \cdot \hat{A}(c-b)] \frac{[(2 k+b) \cdot \hat{A}(b)]}{b^{2}+2 k \cdot b} \\
&-48\left(k \cdot d^{2} k \cdot d^{\perp} \sum_{\substack{b, c \in\{ \pm d, \pm 2 d\} \\
c-b \in\{ \pm d, \pm 2 d\}}} \frac{1}{b^{2}+2 k \cdot b}\right. \\
& \sum_{\substack{b, c \in\{ \pm d, \pm 2 d\} \\
c-b \in\{ \pm d, \pm 2 d\}}} \frac{1}{c^{2}+2 k \cdot c} \frac{1}{b^{2}+2 k \cdot b}
\end{aligned}
$$


Inserting the allowed values for $b$ and $c$

$$
\begin{aligned}
\dddot{\epsilon}(k) & =24 k_{2}\left(\frac{2}{1+2 k_{1}}+\frac{2}{1-2 k_{1}}+\frac{1}{4\left(1+k_{1}\right)}+\frac{1}{4\left(1-k_{1}\right)}\right) \\
& -48 k_{2}^{3}\left(\frac{1}{2\left(1+2 k_{1}\right)\left(1+k_{1}\right)}+\frac{2}{\left(1+2 k_{1}\right)\left(1-2 k_{1}\right)}+\frac{1}{2\left(1-2 k_{1}\right)\left(1-k_{1}\right)}\right)
\end{aligned}
$$

If (13) were to hold, that is if the above quantity were of the form $-k \cdot \dddot{p}(\lambda)$ for all $k$ with $|k|^{2}=\lambda$ then one would have $\dddot{p}(\lambda)=\mu(\lambda) d^{\perp}$, because the right hand side vanishes for $k_{2}=0$. Therefore

$$
\begin{aligned}
\mu(\lambda)=-24 & \left(\frac{2}{1-2 k_{1}}-\frac{4 k_{2}^{2}}{\left(1+2 k_{1}\right)\left(1-2 k_{1}\right)}-\frac{k_{2}^{2}}{\left(1-2 k_{1}\right)\left(1-k_{1}\right)}\right. \\
& \left.+\frac{2}{1+2 k_{1}}+\frac{1}{4\left(1+k_{1}\right)}+\frac{1}{4\left(1-k_{1}\right)}-\frac{k_{2}^{2}}{\left(1+2 k_{1}\right)\left(1+k_{1}\right)}\right)
\end{aligned}
$$

If (13) were to hold, the right hand side of (14) would have to be constant on the circle $\left\{\left(k_{1}, k_{2}\right) \in \mathbb{R}^{2} \mid k_{1}^{2}+k_{2}^{2}=\lambda\right\}$. Since the right hand side of (14) is a meromorphic function of $f\left(k_{1}, k_{2}\right)$, it would then be constant on the complex quadric

$$
Q_{\lambda}=\left\{\left(k_{1}, k_{2}\right) \in \mathbb{C}^{2} \mid k_{1}^{2}+k_{2}^{2}=\lambda\right\}
$$

On the other hand, $f\left(k_{1}, k_{2}\right)$ has a pole with residue $24\left(1-2 k_{2}^{2}\right)$ along the complex line $L=\left\{\left(k_{1}, k_{2}\right) \in \mathbb{C}^{2} \mid k_{1}=\frac{1}{2}\right\}$. Consequently $\left.f\right|_{Q_{\lambda}}$ is infinite on the points of $Q_{\lambda} \cap L$ different from $\left(\frac{1}{2}, \pm \frac{i}{\sqrt{2}}\right)$. This shows that $\left.f\right|_{Q_{\lambda}}$ cannot be constant unless $Q_{\lambda} \cap L \subset\left\{\left(\frac{1}{2}, \pm \frac{i}{\sqrt{2}}\right)\right\}$. For $\lambda>0$, this is not the case. 


\section{References}

[FKLT] J. Feldman, H. Knörrer, D. Lehmann, E. Trubowitz, Fermi Liquids in Two-Space Dimensions, Constructive Physics V. Rivasseau ed. Springer Lecture Notes in Physics 446, 267-300 (1995).

[O] H. R. Ott, Ten Years of Superconductivity: 1980-1990, Kluwer, Dordrecht, 1993.

[S] B. Simon, Trace ideals and their applications, Cambridge University Press, 1979. 